\title{
Synthesis and Biological Evaluation of 3-Benzylidene-4-chromanone Derivatives as Free Radical Scavengers and $\alpha$-Glucosidase Inhibitors
}

\author{
Koichi Takao,* Marimo Yamashita, Aruki Yashiro, and Yoshiaki Sugita \\ Laboratory of Bioorganic Chemistry, Department of Pharmaceutical and Health Sciences, Faculty of Pharmaceutical \\ Sciences, Josai University; 1-1 Keyaki-dai, Sakado, Saitama 350-0295, Japan. \\ Received April 11, 2016; accepted April 25, 2016
}

\begin{abstract}
A series of 3-benzylidene-4-chromanone derivatives (3-20) were synthesized and the structure-activity relationships for antioxidant and $\alpha$-glucosidase inhibitory activities were evaluated. Among synthesized compounds, compounds 5, 13, 18, which contain catechol moiety, showed the potent 1,1-diphenyl-2-picrylhydrazyl (DPPH) free radical scavenging activity (5: $\left.\mathrm{EC}_{50} 13 \mu \mathrm{M} ; 13: \mathrm{EC}_{50} 14 \mu \mathrm{M} ; 18: \mathrm{EC}_{50} 13 \mu \mathrm{M}\right)$. The compounds 12, 14, 18 showed higher $\alpha$-glucosidase inhibitory activity (12: IC $\left.\mathrm{IC}_{50} 15 \mu \mathrm{M} ; 14: \mathrm{IC}_{50} 25 \mu \mathrm{M} ; 18: \mathrm{IC}_{50} 28 \mu \mathrm{M}\right)$. The compound 18 showed both of potent DPPH radical scavenging and $\alpha$-glucosidase inhibitory activities. These data suggest that 3-benzylidene-4-chromanone derivatives, such as compound 18, may serve as the lead compound for the development of novel $\alpha$-glucosidase inhibitors with antioxidant activity.
\end{abstract} ship

Key words 3-benzylidene-4-chromanone; $\alpha$-glucosidase inhibitor; antioxidant; structure-activity relation-

Diabetes mellitus is a chronic metabolic disorder characterized by hyperglycemia resulting from defects in the secretion or action of endogenous insulin, and this hyperglycemia is associated with numerous complications, such as atherosclerosis, cardiac dysfunction, retinopathy, neuropathy, and nephropathy. Thus, one therapeutic approach in diabetes is to reduce the demand for insulin by lowering the corresponding postprandial hyperglycemic levels. One effective approach is to use $\alpha$-glucosidase inhibitors, since $\alpha$-glucosidase is a known therapeutic target for the modulation of postprandial hyperglycemia. $^{1-3)}$ On the other hand, antioxidants are important in the prevention of human diseases. Accumulated evidence suggests that diabetic patients are under oxidative stress, and oxidative stress plays a major role in the pathogenesis of diabetes mellitus. Recently, several researchers have evaluated $\alpha$-glucosidase inhibitors exhibiting free-radical scavenging activity. ${ }^{4-9)}$

(3E)-3-Benzylidene-2,3-dihydro-4H-1-benzopyran-4-ones (3-benzylidene-4-chromanones) constitute a small class of natural products primarily isolated from Hyacinthaceae and Caesalpinioideae. ${ }^{10-14)}$ Several natural and synthetic 3-benzylidene-4-chromanones are related structurally to flavonoids and were found to possess various biological properties such as antioxidant, antifungal, antiviral, anti-mutagenic, antiproliferative, anti-allergic, antihistaminic, anti-inflammatory, and monoamine oxidase inhibitory activity. ${ }^{15-24)}$ However, no systematically evaluated data are available on the inhibitory activity of $\alpha$-glucosidase by 3-benzylidene-4-chromanone derivatives. $^{14)}$

In order to further explore new biological activities of this family of compounds, we synthesized a series of 3-benzylidene-4-chromanone derivatives and investigated the structure-activity relationships (SAR) of these 3-benzylidene4-chromanone derivatives to inhibit $\alpha$-glucosidase and exhibit antioxidant activity.

\section{Results and Discussion}

Chemistry The 3-benzylidene-4-chromanone derivatives presented in this study were synthesized by base-catalyzed condensation of 4-chromanone derivatives with benzaldehyde derivatives according to the method of Siddaiah et al., with small modifications $^{20)}$ (Chart 1). This required the preparation of the 4-chromanone derivatives $\mathbf{1 a}, \mathbf{b}$ and $\mathbf{c}\left(\mathrm{R}^{1}=\mathrm{H}, \mathrm{OH}\right.$ and $\mathrm{OMe}$, respectively). Compound $\mathbf{1 a}$ was commercially available and compounds $\mathbf{1 b}$ and $\mathbf{c}$ were synthesized from resorcinol according to the procedure of Foroumadi et al. $^{21)}$ With $1 \mathbf{1 a}-\mathbf{c}$ in hand, each was condensed with benzaldehyde derivatives $(\mathbf{2} \mathbf{a}-\mathbf{h})$ in the presence of piperidine to provide the 3-benzylidene-4-chromanone derivatives (3-20).

Biological Activity All the synthesized 3-benzylidene4-chromanone derivatives shared similar chemical features and functional groups, such as the presence of hydroxyl and methoxy groups, and were evaluated for their potential 1,1-diphenyl-2-picrylhydrazyl (DPPH) free radical scavenging and $\alpha$-glucosidase inhibitory activities. As shown in Table 1, modification of the 3-benzylidene-4-chromanones on the chromanone ring (A-ring) and the phenyl group (B-ring) of the benzylidene moiety revealed some interesting SAR.

Introduction of the methoxy, monohydroxyl or dimethylamino group, or a halogen atom ( $\mathrm{F}$ or $\mathrm{Cl}$ ) substituent, on the B-ring did not result in DPPH radical scavenging activity, whereas introduction of the 3',4'-dihydroxyl (catechol) group resulted in potent activity (compounds $\mathbf{5}, \mathbf{1 3}, \mathbf{1 8}$, with $\mathrm{EC}_{50}$ values $13,14,13 \mu \mathrm{M}$, respectively). These findings were consistent with the previous reports that showed compound $\mathbf{1 3}^{\mathbf{2 0}}$ and $18^{21)}$ had a strong DPPH radical scavenging activity. The catechol group in the B-ring of 3-benzylidene-4-chromanones is known to be an important structural feature for antioxidant activity. ${ }^{20,21)}$ It was noted that the introduction of a hydroxyl group at the 7-position on the A-ring did not increase the DPPH radical scavenging activity of 3-benzylidene-4-chromanones.

The $\alpha$-glucosidase inhibitory activities of the 3-benzylidene-4-chromanones were determined (Table 1), and 7 of the 18 compounds (compounds $4,5,6, \mathbf{1 2}, \mathbf{1 3}, \mathbf{1 4}, \mathbf{1 8}$ ) were

* To whom correspondence should be addressed. e-mail: ktakao@josai.ac.jp 

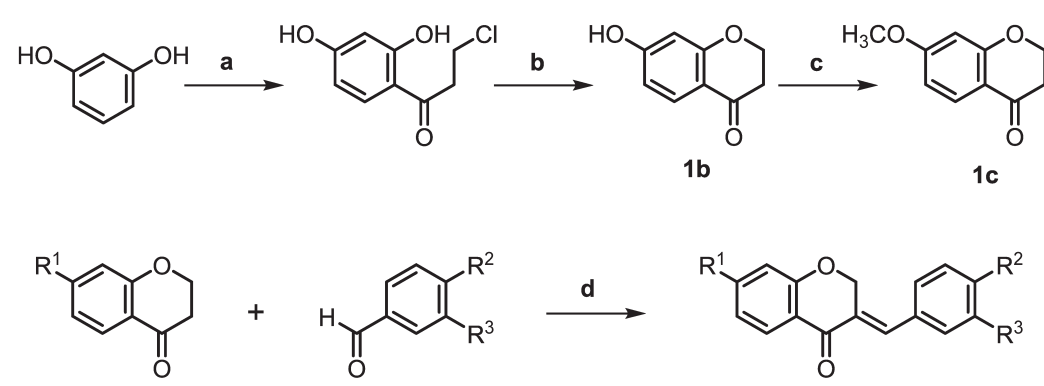

$$
\begin{array}{ll}
\text { 1a: } R^{1}=H & \text { 2a: } R^{2}, R^{3}=H \\
\text { 1b: } R^{1}=O H & \text { 2b: } R^{2}=\mathrm{OH}, R^{3}=H \\
\text { 1c: } R^{1}=\mathrm{OCH}_{3} & \text { 2c: } R^{2}, R^{3}=\mathrm{OH} \\
& \text { 2d: } R^{2}=\mathrm{OCH}_{3}, R^{3}=\mathrm{H} \\
& \text { 2e: } R^{2}, \mathrm{R}^{3}=\mathrm{OCH}_{3} \\
& \text { 2f: } \mathrm{R}^{2}=\mathrm{N}\left(\mathrm{CH}_{3}\right)_{2}, \mathrm{R}^{3}=\mathrm{H} \\
& \text { 2g: } \mathrm{R}^{2}=\mathrm{F}, \mathrm{R}^{3}=\mathrm{H} \\
& \text { 2h: } \mathrm{R}^{2}=\mathrm{Cl}, \mathrm{R}^{3}=\mathrm{H}
\end{array}
$$$$
\mathrm{R}^{1}, \mathrm{R}^{2}, \mathrm{R}^{3}=\mathrm{H}
$$$$
\text { 4: } \mathrm{R}^{1}, \mathrm{R}^{3}=\mathrm{H}, \mathrm{R}^{2}=\mathrm{OH}
$$$$
\text { 5: } \mathrm{R}^{1}=\mathrm{H}, \mathrm{R}^{2}, \mathrm{R}^{3}=\mathrm{OH}
$$$$
\text { 6: } \mathrm{R}^{1}, \mathrm{R}^{3}=\mathrm{H}, \mathrm{R}^{2}=\mathrm{OCH}_{3}
$$$$
\text { 7: } \mathrm{R}^{1}=\mathrm{H}, \mathrm{R}^{2}, \mathrm{R}^{3}=\mathrm{OCH}_{3}
$$$$
\text { 8: } R^{1}, R^{3}=H, R^{2}=N\left(C_{3}\right)_{2}
$$$$
\text { 9: } R^{1}, R^{3}=H, R^{2}=F
$$$$
\text { 10: } R^{1}, R^{3}=H, R^{2}=\mathrm{Cl}
$$$$
\text { 11: } \mathrm{R}^{1}=\mathrm{OH}, \mathrm{R}^{2}, \mathrm{R}^{3}=\mathrm{H}
$$$$
\text { 12: } R^{1}, R^{2}=O H, R^{3}=H
$$$$
\text { 13: } R^{1}, R^{2}, R^{3}=\mathrm{OH}
$$$$
\text { 14: } \mathrm{R}^{1}=\mathrm{OH}, \mathrm{R}^{2}=\mathrm{OCH}_{3}, \mathrm{R}^{3}=\mathrm{H}
$$

\begin{tabular}{|c|c|c|c|c|c|}
\hline Compd & $\mathrm{R}^{1}$ & $\mathrm{R}^{2}$ & $\mathrm{R}^{3}$ & DPPH radical scavenging activity $\mathrm{EC}_{50}(\mu \mathrm{M})$ & $\alpha$-Glucosidase inhibitory activity $\mathrm{IC}_{50}(\mu \mathrm{M})$ \\
\hline 3 & $\mathrm{H}$ & $\mathrm{H}$ & $\mathrm{H}$ & $>200$ & $>100$ \\
\hline 4 & $\mathrm{H}$ & $\mathrm{OH}$ & $\mathrm{H}$ & $>200$ & 79 \\
\hline 5 & $\mathrm{H}$ & $\mathrm{OH}$ & $\mathrm{OH}$ & 13 & 47 \\
\hline 6 & $\mathrm{H}$ & $\mathrm{OCH}_{3}$ & $\mathrm{H}$ & $>200$ & 93 \\
\hline 7 & $\mathrm{H}$ & $\mathrm{OCH}_{3}$ & $\mathrm{OCH}_{3}$ & $>200$ & $>100$ \\
\hline 8 & $\mathrm{H}$ & $\mathrm{N}\left(\mathrm{CH}_{3}\right)_{2}$ & $\mathrm{H}$ & $>200$ & $>100$ \\
\hline 9 & $\mathrm{H}$ & $\mathrm{F}$ & $\mathrm{H}$ & $>200$ & $>100$ \\
\hline 10 & $\mathrm{H}$ & $\mathrm{Cl}$ & $\mathrm{H}$ & $>200$ & $>100$ \\
\hline 11 & $\mathrm{OH}$ & $\mathrm{H}$ & $\mathrm{H}$ & $>200$ & $>100$ \\
\hline 12 & $\mathrm{OH}$ & $\mathrm{OH}$ & $\mathrm{H}$ & $>200$ & 15 \\
\hline 13 & $\mathrm{OH}$ & $\mathrm{OH}$ & $\mathrm{OH}$ & 14 & 48 \\
\hline 14 & $\mathrm{OH}$ & $\mathrm{OCH}_{3}$ & $\mathrm{H}$ & $>200$ & 25 \\
\hline 15 & $\mathrm{OH}$ & $\mathrm{N}\left(\mathrm{CH}_{3}\right)_{2}$ & $\mathrm{H}$ & $>200$ & $>100$ \\
\hline 16 & $\mathrm{OCH}_{3}$ & $\mathrm{H}$ & $\mathrm{H}$ & $>200$ & $>100$ \\
\hline 17 & $\mathrm{OCH}_{3}$ & $\mathrm{OH}$ & $\mathrm{H}$ & $>200$ & $>100$ \\
\hline 18 & $\mathrm{OCH}_{3}$ & $\mathrm{OH}$ & $\mathrm{OH}$ & 13 & 28 \\
\hline 19 & $\mathrm{OCH}_{3}$ & $\mathrm{OCH}_{3}$ & $\mathrm{H}$ & $>200$ & $>100$ \\
\hline 20 & $\mathrm{OCH}_{3}$ & $\mathrm{~N}\left(\mathrm{CH}_{3}\right)_{2}$ & $\mathrm{H}$ & $>200$ & $>100$ \\
\hline Ascorbic acid & & & & 12 & \\
\hline Acarbose & & & & & 900 \\
\hline
\end{tabular}$$
\text { 15: } \mathrm{R}^{1}=\mathrm{OH}, \mathrm{R}^{2}=\mathrm{N}\left(\mathrm{CH}_{3}\right)_{2}, \mathrm{R}^{3}=\mathrm{H}
$$$$
\text { 16: } R^{1}=\mathrm{OCH}_{3}, \mathrm{R}^{2}, \mathrm{R}^{3}=\mathrm{H}
$$$$
\text { 17: } \mathrm{R}^{1}=\mathrm{OCH}_{3}, \mathrm{R}^{2}=\mathrm{OH}, \mathrm{R}^{3}=\mathrm{H}
$$$$
\text { 18: } \mathrm{R}^{1}=\mathrm{OCH}_{3}, \mathrm{R}^{2}, \mathrm{R}^{3}=\mathrm{OH}
$$$$
\text { 19: } R^{1}, R^{2}=\mathrm{OCH}_{3} \mathrm{R}^{3}=\mathrm{H}
$$$$
\text { 20: } \mathrm{R}^{1}=\mathrm{OCH}_{3}, \mathrm{R}^{2}=\mathrm{N}\left(\mathrm{CH}_{3}\right)_{2}, \mathrm{R}^{3}=\mathrm{H}
$$

Reagents and conditions: (a) 3-Chloropropionic acid, $\mathrm{CF}_{3} \mathrm{SO}_{3} \mathrm{H}, 80^{\circ} \mathrm{C}, 1 \mathrm{~h}, 58 \%$; (b) $2 \mathrm{M} \mathrm{NaOH}, 0^{\circ} \mathrm{C}$-r.t. $2 \mathrm{~h}, 43 \%$; (c) iodomethane, $\mathrm{K}_{2} \mathrm{CO}_{3}, \mathrm{DMF}^{\circ} 80^{\circ} \mathrm{C}, 1 \mathrm{~h}, 95 \%$; (d) piperidine, $100^{\circ} \mathrm{C}, 2 \mathrm{~h}$

Chart 1. Synthetic Protocol of 3-Benzylidene-4-chromanone Derivatives 3-20

Table 1. DPPH Free Radical Scavenging and $\alpha$-Glucosidase Inhibitory Activities of 3-Benzylidene-4-chromanone Derivatives

found to have better inhibitory $\alpha$-glucosidase inhibitory activity than a commercial anti-hyperglycemic drug, acarbose $\left(\mathrm{IC}_{50}=900 \mu \mathrm{M}\right)$. Compound $\mathbf{1 2}$ was the most potent inhibitor $\left(\mathrm{IC}_{50}=15 \mu \mathrm{M}\right)$. It was noted that the introduction of a hydroxyl group at the 7-position on the A-ring increased the activity (compounds 12, 14), while the introduction of a methoxy group on the A-ring decreased the activity (compounds 17, 19), except for catechol-substituted compounds (compounds 
13, 18). Consequently, catechol substitution on the B-ring may play an important role in $\alpha$-glucosidase inhibitory activity (compounds 5, 13, 18). These results indicate that hydroxylation of the A- and B-rings is important for $\alpha$-glucosidase inhibitory activity. It has been reported that some flavonoids and polyphenols, as well as sugar derivatives, are effective inhibitors of $\alpha$-glucosidase activity, ${ }^{4,25,26)}$ suggesting that the polyphenols present in 3-benzylidene-4-chromanones are important for $\alpha$-glucosidase inhibitory activity.

Interestingly, compound $\mathbf{1 8}$ showed both potent DPPH free radical scavenging and $\alpha$-glucosidase inhibitory activities. Accumulated evidence suggests that oxidative stress is involved in the pathogenesis of diabetes mellitus and further exacerbates diabetic complications. 3-Benzylidene-4-chromanone derivatives such as compound $\mathbf{1 8}$ may serve as lead compounds for novel $\alpha$-glucosidase inhibitors.

\section{Conclusion}

We synthesized a series of 3-benzylidene-4-chromanone derivatives (3-20) and evaluated their potential for DPPH free radical scavenging and $\alpha$-glucosidase inhibitory activity. As a result, compounds $5, \mathbf{1 3}, \mathbf{1 8}$, which possess a catechol moiety on the B-ring, were found to exhibit potent DPPH free radical scavenging and $\alpha$-glucosidase inhibitory activities. This result indicates that introduction of the $3^{\prime}, 4^{\prime}$-dihydroxyl (catechol) group on the phenyl group of the benzylidene moiety is important for $\alpha$-glucosidase inhibition and antioxidant activities.

This is the first report identifying both DPPH free radical scavenging and $\alpha$-glucosidase inhibitory activities by a 3 -benzylidene-4-chromanone derivative. These results suggest that 3-benzylidene-4-chromanone derivatives such as compound 18 may serve as lead compounds for the development of novel $\alpha$-glucosidase inhibitors.

\section{Experimental}

Chemistry All reagents and solvents were purchased from commercial sources. Analytical TLC was performed on silica-coated plates (silica gel 60F-254, Merck) and visualized under UV light. Column chromatography was carried out using silica gel (Wakogel C-200, Wako Pure Chemical Industries, Ltd., Osaka, Japan). All melting points were determined using a Yanagimoto micro-hot stage and are uncorrected. ${ }^{1} \mathrm{H}-\mathrm{NMR}$ and ${ }^{13} \mathrm{C}-\mathrm{NMR}$ spectra were recorded on a Varian $400-\mathrm{MR} 400 \mathrm{MHz}$ spectrometer using tetramethylsilane as the internal standard. MS spectra were measured using a JEOL JMS-700 spectrometer. Elemental analyses were carried out on a Yanaco CHN MT-6 elemental analyzer.

Synthesis of 4-Chromanones 4-Chromanones $\mathbf{1 b}$ and $\mathbf{c}$ were synthesized according to the previous procedure of Foroumadi et al. $^{21)}$ The products $(\mathbf{1 b}, \mathbf{c})$ were identified by their melting points (mp) and ${ }^{1} \mathrm{H}-\mathrm{NMR}$ spectra.

2,3-Dihydro-7-hydroxy-4H-1-benzopyran-4-one (1b)

Yield $43 \%$. White solid. $\mathrm{mp} 144^{\circ} \mathrm{C}$ (lit. $146-148^{\circ} \mathrm{C}^{20)}$ ). ${ }^{1} \mathrm{H}-\mathrm{NMR}$ (DMSO- $\left.d_{6}, 400 \mathrm{MHz}\right) \delta: 7.59(1 \mathrm{H}, \mathrm{d}, J=8.6 \mathrm{~Hz}, \mathrm{H}-5)$, $6.45(1 \mathrm{H}, \mathrm{dd}, J=8.7,2.3 \mathrm{~Hz}, \mathrm{H}-6), 6.27(1 \mathrm{H}, \mathrm{d}, J=2.2 \mathrm{~Hz}, \mathrm{H}-8)$, $4.43(2 \mathrm{H}, \mathrm{t}, J=6.4 \mathrm{~Hz}, \mathrm{H}-2), 2.64$ (2H, t, $J=6.4 \mathrm{~Hz}, \mathrm{H}-3)$. MS electron ionization (EI) $m / z: 164[\mathrm{M}]^{+}$. The ${ }^{1} \mathrm{H}-\mathrm{NMR}$ spectrum was similar to that previously reported. ${ }^{20)}$

2,3-Dihydro-7-methoxy-4H-1-benzopyran-4-one (1c)

Yield $95 \%$. Pale red solid. mp $44-46^{\circ} \mathrm{C}$ (lit. $46-48^{\circ} \mathrm{C}^{20)}$ ). ${ }^{1} \mathrm{H}-\mathrm{NMR}\left(\mathrm{CDCl}_{3}, 400 \mathrm{MHz}\right) \delta: 7.84(1 \mathrm{H}, \mathrm{d}, J=8.9 \mathrm{~Hz}, \mathrm{H}-5)$,
$6.58(1 \mathrm{H}, \mathrm{dd}, J=8.9,2.6 \mathrm{~Hz}, \mathrm{H}-6), 6.41(1 \mathrm{H}, \mathrm{d}, J=2.3 \mathrm{~Hz}, \mathrm{H}-8)$, $4.52(2 \mathrm{H}, \mathrm{t}, J=6.4 \mathrm{~Hz}, \mathrm{H}-2), 3.84(3 \mathrm{H}, \mathrm{s}, \mathrm{OMe}), 2.76(2 \mathrm{H}, \mathrm{t}$, $J=6.4 \mathrm{~Hz}, \mathrm{H}-3$ ). MS (EI) $m / z: 178[\mathrm{M}]^{+}$. The ${ }^{1} \mathrm{H}-\mathrm{NMR}$ spectrum was similar to that previously reported. ${ }^{20)}$

General Procedure for Preparation of (3E)- 3-Benzylidene-2,3-dihydro-4H-1-benzopyran-4-one Derivatives (3-20) A mixture of the corresponding 4-chromanone (1, $1.0 \mathrm{mmol})$, substituted benzaldehyde $(2,1.2 \mathrm{mmol})$ and piperidine $(1.2 \mathrm{mmol})$ was heated at $100^{\circ} \mathrm{C}$ until complete disappearance of 4-chromanone. After the reaction mixture was diluted with ice-water and acidified with conc. $\mathrm{HCl}$, the whole was extracted with AcOEt. The combined organic layer was washed with brine. The organic layer was dried over $\mathrm{Na}_{2} \mathrm{SO}_{4}$ and the solvent was evaporated under reduced pressure. The residue was purified by silica gel column chromatography (hexane-AcOEt system) to give the corresponding (3E)-3-benzylidene-2,3-dihydro-4H-1-benzopyran-4-one derivatives.

(3E)-2,3-Dihydro-3-(phenylmetylene)-4H-1-benzopyran-4one (3)

Yield $90 \%$. Colorless crystal. $\mathrm{mp} 110^{\circ} \mathrm{C}$ (lit. $111^{\circ} \mathrm{C}^{22)}$ ). ${ }^{1} \mathrm{H}-\mathrm{NMR}\left(\mathrm{CDCl}_{3}, 400 \mathrm{MHz}\right) \delta: 8.03(1 \mathrm{H}, \mathrm{dd}, J=7.9,1.8 \mathrm{~Hz}$, $\mathrm{H}-5), 7.88$ (1H, brs, CH), $7.49(1 \mathrm{H}, \mathrm{ddd}, J=8.4,7.2,1.8 \mathrm{~Hz}$, $\mathrm{H}-7), 7.47-7.40(3 \mathrm{H}, \mathrm{m}, \mathrm{Ph}), 7.33-7.30(2 \mathrm{H}, \mathrm{m}, \mathrm{Ph}), 7.07(1 \mathrm{H}$, ddd, $J=7.9,7.2,1.0 \mathrm{~Hz}, \mathrm{H}-6), 6.97(1 \mathrm{H}, \mathrm{dd}, J=8.4,1.0 \mathrm{~Hz}$, $\mathrm{H}-8), 5.36$ (2H, d, $J=1.9 \mathrm{~Hz}, \mathrm{H}-2)$. MS (EI) $m / z: 236[\mathrm{M}]^{+}$. The ${ }^{1} \mathrm{H}-\mathrm{NMR}$ spectrum was similar to that previously reported. ${ }^{22)}$

(3E)-2,3-Dihydro-3-[(4-hydroxyphenyl)methylene]-4H-1benzopyran-4-one (4)

Yield $84 \%$. Yellow crystal. mp $226^{\circ} \mathrm{C}$ (lit. $224-225^{\circ} \mathrm{C}^{27)}$ ). ${ }^{1} \mathrm{H}-\mathrm{NMR}$ (DMSO- $\left.d_{6}, 400 \mathrm{MHz}\right) \delta: 10.12(1 \mathrm{H}, \mathrm{s}, \mathrm{OH}), 7.85$ $(1 \mathrm{H}, \mathrm{dd}, J=7.9,1.8 \mathrm{~Hz}, \mathrm{H}-5), 7.66(1 \mathrm{H}, \mathrm{brs}, \mathrm{CH}), 7.56(1 \mathrm{H}$, ddd, $J=8.3,7.2,1.8 \mathrm{~Hz}, \mathrm{H}-7), 7.33\left(2 \mathrm{H}, \mathrm{d}, J=8.6 \mathrm{~Hz}, \mathrm{H}-2^{\prime}\right.$ and $\left.\mathrm{H}-6^{\prime}\right), 7.10(1 \mathrm{H}$, ddd, J=7.9, 7.2, $1.0 \mathrm{~Hz}, \mathrm{H}-6), 7.03(1 \mathrm{H}, \mathrm{dd}$, $J=8.3,1.0 \mathrm{~Hz}, \mathrm{H}-8), 6.87\left(2 \mathrm{H}, \mathrm{d}, J=8.6 \mathrm{~Hz}, \mathrm{H}-3^{\prime}\right.$ and $\left.\mathrm{H}-5^{\prime}\right)$, $5.41(2 \mathrm{H}, \mathrm{d}, J=1.9 \mathrm{~Hz}, \mathrm{H}-2)$. MS (EI) $m / z: 252[\mathrm{M}]^{+}$. The ${ }^{1} \mathrm{H}-\mathrm{NMR}$ spectrum was similar to that previously reported. ${ }^{27)}$

(3E)-2,3-Dihydro-3-[(3,4-dihydroxyphenyl)methylene]-4H-1benzopyran-4-one (5)

Yield $78 \%$. Yellow crystal. mp $225^{\circ} \mathrm{C}$ (lit. $224-225^{\circ} \mathrm{C}^{23)}$ ). ${ }^{1} \mathrm{H}-\mathrm{NMR}$ (DMSO- $\left.d_{6}, 400 \mathrm{MHz}\right) \delta: 7.84(1 \mathrm{H}, \mathrm{dd}, J=7.9,1.7 \mathrm{~Hz}$, $\mathrm{H}-5), 7.58(1 \mathrm{H}$, brs, CH), $7.56(2 \mathrm{H}, \mathrm{ddd}, J=8.3,7.1,1.7 \mathrm{~Hz}$, $\mathrm{H}-7), 7.10(1 \mathrm{H}$, ddd, $J=7.9,7.1,1.0 \mathrm{~Hz}, \mathrm{H}-6), 7.03(1 \mathrm{H}, \mathrm{dd}$, $J=8.3,1.0 \mathrm{~Hz}, \mathrm{H}-8), 6.86-6.77$ (3H, m, H-2', H-5' and $\left.\mathrm{H}-6^{\prime}\right)$, $5.40(2 \mathrm{H}, \mathrm{d}, J=1.9 \mathrm{~Hz}, \mathrm{H}-2)$. MS (EI) $m / z: 268[\mathrm{M}]^{+}$. The ${ }^{1} \mathrm{H}-\mathrm{NMR}$ spectrum was similar to that previously reported. ${ }^{23)}$

(3E)-2,3-Dihydro-3-[(4-methoxyphenyl)methylene]-4H-1benzopyran-4-one (6)

Yield $80 \%$. Yellow crystal. mp $130-132^{\circ} \mathrm{C}$ (lit. $131-133^{\circ} \mathrm{C}^{27)}$ ). ${ }^{1} \mathrm{H}-\mathrm{NMR}\left(\mathrm{CDCl}_{3}, 400 \mathrm{MHz}\right) \delta: 8.02(1 \mathrm{H}, \mathrm{dd}, J=7.7,1.7 \mathrm{~Hz}$, $\mathrm{H}-5), 7.84(1 \mathrm{H}$, brs, CH), $7.48(1 \mathrm{H}$, ddd, $J=8.3,7.1,1.7 \mathrm{~Hz}$, $\mathrm{H}-7), 7.29\left(2 \mathrm{H}, \mathrm{d}, J=8.6 \mathrm{~Hz}, \mathrm{H}-2^{\prime}\right.$ and $\left.\mathrm{H}-6^{\prime}\right), 7.07$ (1H, ddd, $J=7.7,7.1,1.1 \mathrm{~Hz}, \mathrm{H}-6), 6.98\left(2 \mathrm{H}, \mathrm{d}, J=8.6 \mathrm{~Hz}, \mathrm{H}-3^{\prime}\right.$ and $\left.\mathrm{H}-5^{\prime}\right)$, $6.97(1 \mathrm{H}, \mathrm{dd}, J=8.3,1.1 \mathrm{~Hz}, \mathrm{H}-8), 5.38(2 \mathrm{H}, \mathrm{d}, J=1.7 \mathrm{~Hz}, \mathrm{H}-2)$, $3.87(3 \mathrm{H}, \mathrm{s}, \mathrm{OMe})$. MS (EI) $m / z: 266[\mathrm{M}]^{+}$. The ${ }^{1} \mathrm{H}-\mathrm{NMR}$ spectrum was similar to that previously reported. ${ }^{27)}$

(3E)-2,3-Dihydro-3-[(3,4-dimethoxyphenyl)methylene]$4 H$-1-benzopyran-4-one (7)

Yield $65 \%$. Yellow crystal. mp $123^{\circ} \mathrm{C}$. ${ }^{1} \mathrm{H}-\mathrm{NMR}\left(\mathrm{CDCl}_{3}\right.$, $400 \mathrm{MHz}) \delta: 8.02(1 \mathrm{H}, \mathrm{dd}, J=7.9,1.7 \mathrm{~Hz}, \mathrm{H}-5), 7.83(1 \mathrm{H}$, brs, $\mathrm{CH}), 7.48(1 \mathrm{H}$, ddd, $J=8.3,7.1,1.7 \mathrm{~Hz}, \mathrm{H}-7), 7.08(1 \mathrm{H}$, ddd, $J=7.9,7.1,0.9 \mathrm{~Hz}, \mathrm{H}-6), 6.97(1 \mathrm{H}, \mathrm{dd}, J=8.3,0.9 \mathrm{~Hz}, \mathrm{H}-8)$, 
$6.94\left(1 \mathrm{H}, \mathrm{d}, J=8.3 \mathrm{~Hz}, \mathrm{H}-5^{\prime}\right), 6.90(1 \mathrm{H}, \mathrm{dd}, J=8.3,1.9 \mathrm{~Hz}$, H-6' $\left.{ }^{\prime}\right), 6.87\left(1 \mathrm{H}, \mathrm{d}, J=1.9 \mathrm{~Hz}, \mathrm{H}-2^{\prime}\right), 5.40(2 \mathrm{H}, \mathrm{d}, J=2.9 \mathrm{~Hz}$, $\mathrm{H}-2), 3.94(3 \mathrm{H}, \mathrm{s}, \mathrm{OMe}), 3.92(3 \mathrm{H}, \mathrm{s}, \mathrm{OMe}) .{ }^{13} \mathrm{C}-\mathrm{NMR}\left(\mathrm{CDCl}_{3}\right.$, $100 \mathrm{MHz}) \delta$ : 182.1, 160.9, 150.3, 148.9, 137.5, 135.7, 129.1, 127.9, 127.2, 123.6, 122.0, 121.8, 117.8, 113.2, 111.0, 67.8, 56.0. MS (EI) $m / z: 296[M]^{+}$. Anal. Calcd for $\mathrm{C}_{18} \mathrm{H}_{16} \mathrm{O}_{4}: \mathrm{C}, 72.96 ; \mathrm{H}$, 5.44. Found: C, 72.73; H, 5.28.

(3E)-2,3-Dihydro-3-[(4-dimethylaminophenyl)methylene]4H-1-benzopyran-4-one (8)

Yield $51 \%$. Yellow crystal. mp $149-151^{\circ} \mathrm{C}\left(\right.$ lit. $\left.150-153^{\circ} \mathrm{C}^{17)}\right)$. ${ }^{1} \mathrm{H}-\mathrm{NMR}\left(\mathrm{CDCl}_{3}, 400 \mathrm{MHz}\right) \delta: 8.02(1 \mathrm{H}, \mathrm{dd}, J=8.0,1.7 \mathrm{~Hz}$, $\mathrm{H}-5), 7.83$ (1H, brs, CH), $7.46(1 \mathrm{H}, \mathrm{ddd}, J=8.3,7.1,1.7 \mathrm{~Hz}$, $\mathrm{H}-7), 7.27\left(2 \mathrm{H}, \mathrm{d}, J=8.9 \mathrm{~Hz}, \mathrm{H}-2^{\prime}\right.$ and $\left.\mathrm{H}-6^{\prime}\right), 7.05(1 \mathrm{H}$, ddd, $J=8.0,7.11 .0 \mathrm{~Hz}, \mathrm{H}-6), 6.95(1 \mathrm{H}, \mathrm{dd}, J=8.3,1.0 \mathrm{~Hz}, \mathrm{H}-8), 6.73$ $\left(2 \mathrm{H}, \mathrm{d}, J=8.9 \mathrm{~Hz}, \mathrm{H}-3^{\prime}\right.$ and $\left.\mathrm{H}-5^{\prime}\right), 5.44(2 \mathrm{H}, \mathrm{d}, J=2.0 \mathrm{~Hz}, \mathrm{H}-2)$, $3.05\left(6 \mathrm{H}, \mathrm{s}, \mathrm{NMe}_{2}\right)$. MS (EI) $m / z: 279[\mathrm{M}]^{+}$. The ${ }^{1} \mathrm{H}-\mathrm{NMR}$ spectrum was similar to that previously reported. ${ }^{17)}$

$(3 E)$-2,3-Dihydro-3-[(4-fluorophenyl)methylene]-4H-1benzopyran-4-one (9)

Yield $36 \%$. White crystal. mp $150{ }^{\circ} \mathrm{C}$. ${ }^{1} \mathrm{H}-\mathrm{NMR}\left(\mathrm{CDCl}_{3}\right.$, $400 \mathrm{MHz}) \delta: 8.03(1 \mathrm{H}, \mathrm{dd}, J=8.0,1.7 \mathrm{~Hz}, \mathrm{H}-5), 7.83(1 \mathrm{H}, \mathrm{brs}$, $\mathrm{CH}), 7.50$ (1H, ddd, $J=8.3,7.1,1.7 \mathrm{~Hz}, \mathrm{H}-7), 7.33-7.29(2 \mathrm{H}, \mathrm{m}$, $\mathrm{H}-2^{\prime}$ and $\left.\mathrm{H}-6^{\prime}\right), 7.18-7.12\left(2 \mathrm{H}, \mathrm{m}, \mathrm{H}-3^{\prime}\right.$ and $\left.\mathrm{H}-5^{\prime}\right), 7.09(1 \mathrm{H}$, ddd, $J=8.0,7.1,0.9 \mathrm{~Hz}, \mathrm{H}-6), 6.98(1 \mathrm{H}, \mathrm{dd}, J=8.3,0.9 \mathrm{~Hz}$, $\mathrm{H}-8), 5.33$ (2H, d, $J=1.7 \mathrm{~Hz}, \mathrm{H}-2)$. MS (EI) $m / z$ : $254[\mathrm{M}]^{+}$. The ${ }^{1} \mathrm{H}-\mathrm{NMR}$ spectrum was similar to that previously reported. ${ }^{28)}$

(3E)-3-[(4-Chlorophenyl)methylene]-2,3-dihydro-4H-1benzopyran-4-one (10)

Yield $70 \%$. White crystal. mp $172^{\circ} \mathrm{C}$ (lit. $170-173^{\circ} \mathrm{C}^{27)}$ ). ${ }^{1} \mathrm{H}-\mathrm{NMR}\left(\mathrm{CDCl}_{3}, 400 \mathrm{MHz}\right) \delta: 8.02(1 \mathrm{H}, \mathrm{dd}, J=7.7,1.7 \mathrm{~Hz}$, $\mathrm{H}-5), 7.81(1 \mathrm{H}$, brs, CH), $7.50(1 \mathrm{H}, \mathrm{ddd}, J=8.3,7.1,1.7 \mathrm{~Hz}$, $\mathrm{H}-7), 7.43\left(2 \mathrm{H}, \mathrm{d}, J=8.6 \mathrm{~Hz}, \mathrm{H}-2^{\prime}\right.$ and $\left.\mathrm{H}-6^{\prime}\right), 7.25(2 \mathrm{H}, \mathrm{d}$, $J=8.6 \mathrm{~Hz}, \mathrm{H}-3^{\prime}$ and $\left.\mathrm{H}-5^{\prime}\right), 7.09(1 \mathrm{H}$, ddd, $J=7.7,7.1,0.9 \mathrm{~Hz}$, $\mathrm{H}-6), 6.98(1 \mathrm{H}, \mathrm{dd}, J=8.3,0.9 \mathrm{~Hz}, \mathrm{H}-8), 5.32(2 \mathrm{H}, \mathrm{d}, J=2.0 \mathrm{~Hz}$, $\mathrm{H}-2$ ). MS (EI) $m / z: 270[\mathrm{M}]^{+}$. The ${ }^{1} \mathrm{H}-\mathrm{NMR}$ spectrum was similar to that previously reported. ${ }^{27)}$

(3E)-2,3-Dihydro-7-hydroxy-3-(phenylmethylene)-4H-1benzopyran-4-one (11)

Yield 98\%. Colorless crystal. mp $192-194^{\circ} \mathrm{C} .{ }^{1} \mathrm{H}-\mathrm{NMR}$ $\left(\mathrm{DMSO}_{6}, 400 \mathrm{MHz}\right) \delta: 7.90(1 \mathrm{H}, \mathrm{d}, J=8.6 \mathrm{~Hz}, \mathrm{H}-5), 7.83(1 \mathrm{H}$, brs, CH), 7.66-7.55 (5H, m, Ph), $6.70(1 \mathrm{H}, \mathrm{dd}, J=8.6,2.3 \mathrm{~Hz}$, H-6), $6.46(1 \mathrm{H}, \mathrm{d}, J=2.3 \mathrm{~Hz}, \mathrm{H}-8), 5.49(2 \mathrm{H}, \mathrm{d}, J=2.0 \mathrm{~Hz}$, $\mathrm{H}-2$ ). MS (EI) $m / z$ : $252[\mathrm{M}]^{+}$. The ${ }^{1} \mathrm{H}-\mathrm{NMR}$ spectrum was similar to that previously reported. ${ }^{19)}$

(3E)-2,3-Dihydro-7-hydroxy-3 - [(4-hydroxy phenyl)methylene]-4H-1-benzopyran-4-one (12)

Yield $69 \%$. Yellow crystal. mp $263^{\circ} \mathrm{C}$ (lit. $220-222^{\circ} \mathrm{C}^{20)}$ ). ${ }^{1} \mathrm{H}-\mathrm{NMR}$ (DMSO- $\left.d_{6}, 400 \mathrm{MHz}\right) \delta$ : $7.73(1 \mathrm{H}, \mathrm{d}, J=8.6 \mathrm{~Hz}$, $\mathrm{H}-5), 7.60\left(1 \mathrm{H}\right.$, brs, CH), $7.30\left(2 \mathrm{H}, \mathrm{d}, J=8.6 \mathrm{~Hz}, \mathrm{H}-2^{\prime}\right.$ and H-6' $), 6.87\left(2 \mathrm{H}, \mathrm{d}, J=8.6 \mathrm{~Hz}, \mathrm{H}-3^{\prime}\right.$ and $\left.\mathrm{H}-5^{\prime}\right), 6.54(1 \mathrm{H}, \mathrm{dd}$, $J=8.6,2.2 \mathrm{~Hz}, \mathrm{H}-6), 6.31(1 \mathrm{H}, \mathrm{d}, J=2.2 \mathrm{~Hz}, \mathrm{H}-8), 5.35(2 \mathrm{H}, \mathrm{d}$, $J=1.7 \mathrm{~Hz}, \mathrm{H}-2$ ). MS (EI) $m / z: 268[\mathrm{M}]^{+}$. The ${ }^{1} \mathrm{H}-\mathrm{NMR}$ spectrum was similar to that previously reported. ${ }^{20)}$

(3E)-2,3-Dihydro-3-[(3,4-dihydroxyphenyl)methylene]-7hydroxy-4H-1-benzopyran-4-one (13)

Yield $36 \%$. Yellow crystal. mp $239^{\circ} \mathrm{C}$ (lit. $220-222^{\circ} \mathrm{C}^{20)}$ ). ${ }^{1} \mathrm{H}-\mathrm{NMR}$ (DMSO- $\left.d_{6}, 400 \mathrm{MHz}\right) \delta: 7.72(1 \mathrm{H}, \mathrm{d}, J=8.9 \mathrm{~Hz}, \mathrm{H}-5)$, $7.52\left(1 \mathrm{H}\right.$, brs, CH), 6.85-6.81 (2H, m, H-2' and $\left.\mathrm{H}-5^{\prime}\right), 6.76$ $\left(1 \mathrm{H}, \mathrm{dd}, J=8.3,2.0 \mathrm{~Hz}, \mathrm{H}-6^{\prime}\right), 6.54(1 \mathrm{H}, \mathrm{dd}, J=8.8,2.3 \mathrm{~Hz}$, $\mathrm{H}-5), 6.31$ (1H, d, $J=2.3 \mathrm{~Hz}, \mathrm{H}-8), 5.35$ (2H, d, $J=1.4 \mathrm{~Hz}, \mathrm{H}-2)$. MS (EI) $m / z: 284[\mathrm{M}]^{+}$. The ${ }^{1} \mathrm{H}-\mathrm{NMR}$ spectrum was similar to that previously reported. ${ }^{20)}$

(3E)-2,3-Dihydro-7-hydroxy-3-[(4-methoxy phenyl)methylene]-4H-1-benzopyran-4-one (14)

Yield $84 \%$. Yellow crystal. mp $223^{\circ} \mathrm{C}$ (lit. $206-208^{\circ} \mathrm{C}^{20)}$ ). ${ }^{1} \mathrm{H}-\mathrm{NMR}\left(\mathrm{DMSO}-d_{6}, 400 \mathrm{MHz}\right) \delta: 7.72(1 \mathrm{H}, \mathrm{d}, J=8.7 \mathrm{~Hz}, \mathrm{H}-5)$, $7.64(1 \mathrm{H}, \mathrm{brs}, \mathrm{CH}), 7.40\left(2 \mathrm{H}, \mathrm{d}, J=8.8 \mathrm{~Hz}, \mathrm{H}-2^{\prime}\right.$ and $\left.\mathrm{H}-6^{\prime}\right), 7.05$ $\left(2 \mathrm{H}, \mathrm{d}, J=8.8 \mathrm{~Hz}, \mathrm{H}-3^{\prime}\right.$ and $\left.\mathrm{H}-5^{\prime}\right), 6.54(1 \mathrm{H}, \mathrm{dd}, J=8.7,2.2 \mathrm{~Hz}$, H-6), 6.30 (1H, d, $J=2.2 \mathrm{~Hz}, \mathrm{H}-8), 5.35(2 \mathrm{H}, \mathrm{d}, J=1.9 \mathrm{~Hz}, \mathrm{H}-2)$, $3.82(3 \mathrm{H}, \mathrm{s}, \mathrm{OMe})$. MS (EI) $m / z: 282[\mathrm{M}]^{+}$. The ${ }^{1} \mathrm{H}-\mathrm{NMR}$ spectrum was similar to that previously reported. ${ }^{20)}$

(3E)-2,3-Dihydro-3-[(4-dimethylaminophenyl)methylene]-7hydroxy-4H-1-benzopyran-4-one (15)

Yield $78 \%$. Yellow crystal. mp $266^{\circ} \mathrm{C}$ (lit. $264-266^{\circ} \mathrm{C}^{20)}$ ). ${ }^{1} \mathrm{H}-\mathrm{NMR}$ (DMSO- $\left.d_{6}, 400 \mathrm{MHz}\right) \delta: 7.72(1 \mathrm{H}, \mathrm{d}, J=8.8 \mathrm{~Hz}, \mathrm{H}-5)$, 7.59 (1H, brs, CH), $7.30\left(2 \mathrm{H}, \mathrm{d}, J=8.9 \mathrm{~Hz}, \mathrm{H}-2^{\prime}\right.$ and $\left.\mathrm{H}-6^{\prime}\right), 6.78$ $\left(2 \mathrm{H}, \mathrm{d}, J=8.9 \mathrm{~Hz}, \mathrm{H}-3^{\prime}\right.$ and $\left.\mathrm{H}-5^{\prime}\right), 6.53(1 \mathrm{H}, \mathrm{dd}, J=8.8,2.3 \mathrm{~Hz}$, H-6), 6.31 (1H, d, $J=2.3 \mathrm{~Hz}, \mathrm{H}-8), 5.38$ (2H, d, $J=1.7 \mathrm{~Hz}, \mathrm{H}-2)$, $3.00\left(6 \mathrm{H}, \mathrm{s}, \mathrm{NMe}_{2}\right)$. MS (EI) $m / z: 295[\mathrm{M}]^{+}$. The ${ }^{1} \mathrm{H}-\mathrm{NMR}$ spectrum was similar to that previously reported. ${ }^{20)}$

(3E)-2,3-Dihydro-7-methoxy-3-(phenylmethylene)-4H-1benzopyran-4-one (16)

Yield $62 \%$. Yellow solid. mp $69^{\circ} \mathrm{C}$ (lit. $\left.94^{\circ} \mathrm{C}^{29}\right)$ ). ${ }^{1} \mathrm{H}-\mathrm{NMR}$ $\left(\mathrm{CDCl}_{3}, 400 \mathrm{MHz}\right) \delta: 7.97(1 \mathrm{H}, \mathrm{d}, J=8.8 \mathrm{~Hz}, \mathrm{H}-5), 7.85(1 \mathrm{H}$, brs, $\mathrm{CH}), 7.46-7.38$ (3H, m, H-3', $\mathrm{H}-4^{\prime}$ and $\left.\mathrm{H}-5^{\prime}\right), 7.32-7.28$ $\left(2 \mathrm{H}, \mathrm{m}, \mathrm{H}-2^{\prime}\right.$ and $\left.\mathrm{H}-6^{\prime}\right), 6.63(1 \mathrm{H}, \mathrm{dd}, J=8.8,2.3 \mathrm{~Hz}, \mathrm{H}-6)$, $6.40(1 \mathrm{H}, \mathrm{d}, J=2.3 \mathrm{~Hz}, \mathrm{H}-8), 5.33(2 \mathrm{H}, \mathrm{d}, J=2.0 \mathrm{~Hz}, \mathrm{H}-2), 3.84$ $(3 \mathrm{H}, \mathrm{s}, \mathrm{OMe}) .{ }^{13} \mathrm{C}-\mathrm{NMR}\left(\mathrm{CDCl}_{3}, 100 \mathrm{MHz}\right) \delta$ : 181.0, 166.0, $163.1,136.8,134.5,130.8,129.9,129.7,129.2,128.7,115.7$, 110.5, 100.7, 67.8, 55.6. MS (EI) $m / z: 266[\mathrm{M}]^{+}$. The ${ }^{1} \mathrm{H}-\mathrm{NMR}$ spectrum was similar to that previously reported. ${ }^{29)}$

(3E)-2,3-Dihydro-3-[(4-hydroxyphenyl)methylene]-7methoxy-4H-1-benzopyran-4-one (17)

Yield 74\%. Yellow crystal. mp $226^{\circ} \mathrm{C} .{ }^{1} \mathrm{H}-\mathrm{NMR}$ (DMSO- $d_{6}$, $400 \mathrm{MHz}) \delta: 7.80(1 \mathrm{H}, \mathrm{d}, J=8.9 \mathrm{~Hz}, \mathrm{H}-5), 7.63(1 \mathrm{H}$, brs, CH), $7.32\left(2 \mathrm{H}, \mathrm{d}, J=8.8 \mathrm{~Hz}, \mathrm{H}-2^{\prime}\right.$ and $\left.\mathrm{H}-6^{\prime}\right), 6.87(2 \mathrm{H}, \mathrm{d}, J=8.8 \mathrm{~Hz}$, H-3' and H-5'), $6.69(1 \mathrm{H}, \mathrm{dd}, J=8.9,2.3 \mathrm{~Hz}, \mathrm{H}-6), 6.56(1 \mathrm{H}$, d, $J=2.3 \mathrm{~Hz}, \mathrm{H}-8), 5.41(2 \mathrm{H}, \mathrm{dd}, J=1.8 \mathrm{~Hz}, \mathrm{H}-2), 3.83(3 \mathrm{H}, \mathrm{s}$, OMe). MS (EI) $m / z: 282[\mathrm{M}]^{+}$. The ${ }^{1} \mathrm{H}-\mathrm{NMR}$ spectrum was similar to that previously reported. ${ }^{30)}$

(3E)-2,3-Dihydro-3-[(3,4-dihydroxyphenyl)methylene]-7methoxy-4H-1-benzopyran-4-one (18)

Yield $81 \%$. Yellow solid. mp $204^{\circ} \mathrm{C} .{ }^{1} \mathrm{H}-\mathrm{NMR}$ (DMSO- $d_{6}$, $400 \mathrm{MHz}) \delta: 9.72(1 \mathrm{H}$, brs, OH), $9.32(1 \mathrm{H}$, brs, OH), $8.86(1 \mathrm{H}$, $\mathrm{d}, J=8.9 \mathrm{~Hz}, \mathrm{H}-5), 7.61(1 \mathrm{H}, \mathrm{br}, \mathrm{CH}), 6.92-6.81$ (3H, m, H-2', H-5' and H-6'), $6.75(1 \mathrm{H}$, dd, $J=8.9,2.3 \mathrm{~Hz}, \mathrm{H}-6), 6.62(1 \mathrm{H}$, d, $J=2.3 \mathrm{~Hz}, \mathrm{H}-8), 5.46$ (2H, d, $J=1.8 \mathrm{~Hz}, \mathrm{H}-2), 3.89$ (3H, s, OMe). ${ }^{13} \mathrm{C}-\mathrm{NMR}$ (DMSO- $\left.d_{6}, 100 \mathrm{MHz}\right) \delta$ : 179.7, 165.5, 162.4, $147.6,145.4,136.5,128.9,127.4,125.4,123.3,117.7,115.9$, 115.3, 110.3, 100.9, 67.8, 55.8. [M] $]^{+}$. HR-MS m/z: Calcd for $\mathrm{C}_{17} \mathrm{H}_{14} \mathrm{O}_{5}\left(\mathrm{M}^{+}\right)$: 298.0843; Found: 298.0841. Anal. Calcd for $\mathrm{C}_{17} \mathrm{H}_{14} \mathrm{O}_{5}$ : C, 68.45; H, 4.73. Found: $\mathrm{C}, 68.05 ; \mathrm{H}, 4.59$.

(3E)-2,3-Dihydro-7-methoxy-3-[(4-methoxy phenyl)methylene]-4H-1-benzopyran-4-one (19)

Yield 68\%. Pale yellow crystal. mp $124-126^{\circ} \mathrm{C}$ (lit. $\left.130-132^{\circ} \mathrm{C}^{20)}\right) .{ }^{1} \mathrm{H}-\mathrm{NMR}\left(\mathrm{CDCl}_{3}, 400 \mathrm{MHz}\right) \delta: 7.96(1 \mathrm{H}, \mathrm{d}$, $J=8.8 \mathrm{~Hz}, \mathrm{H}-5), 7.81(1 \mathrm{H}$, brs, CH), $7.28(2 \mathrm{H}, \mathrm{d}, J=8.9 \mathrm{~Hz}$, $\mathrm{H}-2^{\prime}$ and $\left.\mathrm{H}-6^{\prime}\right), 6.97\left(2 \mathrm{H}, \mathrm{d}, J=8.9 \mathrm{~Hz}, \mathrm{H}-3^{\prime}\right.$ and $\left.\mathrm{H}-5^{\prime}\right), 6.63$ $(1 \mathrm{H}, \mathrm{dd}, J=8.8,2.3 \mathrm{~Hz}, \mathrm{H}-6), 6.40(1 \mathrm{H}, \mathrm{d}, J=2.3 \mathrm{~Hz}, \mathrm{H}-8), 5.37$ $(2 \mathrm{H}, \mathrm{d}, J=2.0 \mathrm{~Hz}, \mathrm{H}-2), 3.86(3 \mathrm{H}, \mathrm{s}, \mathrm{OMe}), 3.85$ (3H, s, OMe). MS (EI) $m / z$ : $296[\mathrm{M}]^{+}$. The ${ }^{1} \mathrm{H}-\mathrm{NMR}$ spectrum was similar to that previously reported. ${ }^{20)}$ 
(3E)-2,3-Dihydro-3-[(4-dimetylaminophenyl)methylene]-7methoxy-4H-1-benzopyran-4-one (20)

Yield 29\%. Yellow crystal. mp $184-186^{\circ} \mathrm{C}$. ${ }^{1} \mathrm{H}-\mathrm{NMR}$ $\left(\mathrm{CDCl}_{3}, 400 \mathrm{MHz}\right) \delta: 7.96(1 \mathrm{H}, \mathrm{d}, J=8.9 \mathrm{~Hz}, \mathrm{H}-5), 7.80(1 \mathrm{H}$, brs, CH), $7.25\left(2 \mathrm{H}, \mathrm{d}, J=8.9 \mathrm{~Hz}, \mathrm{H}-2^{\prime}\right.$ and $\left.\mathrm{H}-6^{\prime}\right), 6.72(2 \mathrm{H}, \mathrm{d}$, $J=8.9 \mathrm{~Hz}, \mathrm{H}-3^{\prime}$ and $\left.\mathrm{H}-5^{\prime}\right), 6.63(1 \mathrm{H}, \mathrm{dd}, J=8.9,2.3 \mathrm{~Hz}, \mathrm{H}-6)$, $6.40(1 \mathrm{H}, \mathrm{d}, J=2.3 \mathrm{~Hz}, \mathrm{H}-8), 5.42(2 \mathrm{H}, \mathrm{d}, J=1.7 \mathrm{~Hz}, \mathrm{H}-2)$, $3.84(3 \mathrm{H}, \mathrm{s}, \mathrm{OMe}), 3.04\left(6 \mathrm{H}, \mathrm{s}, \mathrm{NMe}_{2}\right) .{ }^{13} \mathrm{C}-\mathrm{NMR}\left(\mathrm{CDCl}_{3}\right.$, $100 \mathrm{MHz}) \delta$ : 181.0, 165.6, 162.7, 150.9, 137.5, 132.3, 129.5, 126.1, 122.3, 116.1, 111.7, 110.0, 100.7, 68.4, 55.6, 40.1. MS (EI) m/z: $309[\mathrm{M}]^{+}$. Anal. Calcd for $\mathrm{C}_{19} \mathrm{H}_{19} \mathrm{NO}_{3}$ : C, 73.77; H, 6.19; N, 4.53. Found: C, 73.71; H, 6.14; N, 4.59.

Biological Assay $\alpha$-Glucosidase from Saccharomyces cerevisiae and 4-nitrophenyl $\alpha$-D-glucopyranoside (PNP-G) were purchased from Sigma Chemical Co., St. Louis, MO, U.S.A. DPPH radical was purchased from Tokyo Chemical Industry Co., Tokyo, Japan.

DPPH Radical Scavenging Assay DPPH radical scavenging activity was measured according to the method of Nile et al. ${ }^{31)}$ with minor modifications. Briefly, $180 \mu \mathrm{L}$ of $100 \mu \mathrm{M}$ DPPH solution in MeOH was mixed with $20 \mu \mathrm{L}$ of various concentrations of the sample solution in $\mathrm{MeOH}$. The absorbance of the mixture was measured at $517 \mathrm{~nm}$ using a microplate reader (Molecular Devices SPECTRA MAX 190). The sample solution was replaced by $\mathrm{MeOH}$ as a control. Ascorbic acid was used as a positive control.

$\boldsymbol{\alpha}$-Glucosidase Inhibitory Assay $\alpha$-Glucosidase inhibitory activity was assayed using the method of Takahashi and Miyazawa ${ }^{32)}$ with minor modifications. Briefly, $210 \mu \mathrm{L}$ of $50 \mathrm{~mm}$ phosphate buffer ( $\mathrm{pH} 6.8$ ) containing $100 \mathrm{~mm} \mathrm{NaCl}$, $30 \mu \mathrm{L}$ of $0.25 \mathrm{U} / \mathrm{mL} \alpha$-glucosidase dissolved in the buffer, $30 \mu \mathrm{L}$ of $7 \mathrm{~mm}$ PNP-G as a substrate dissolved in the buffer, and $30 \mu \mathrm{L}$ of various concentrations of samples dissolved in dimethyl sulfoxide (DMSO) were mixed and the increment in absorption at $405 \mathrm{~nm}$, due to the hydrolysis of PNP-G by $\alpha$-glucosidase, was monitored continuously with a micro-plate reader (Molecular Devices SPECTRA MAX 190). The sample solution was replaced by DMSO as a control. Acarbose was used as a positive control.

Conflict of Interest The authors declare no conflict of interest.

\section{References}

1) Grundy S. M., Hansen B., Smith S. C. Jr., Cleeman J. I., Kahn R. A., American Heart Association, National Heart, Lung, and Blood Institute, American Diabetes Association, Circulation, 109, 551-556 (2004).

2) Ahmed N., Diabetes Res. Clin. Pract., 67, 3-21 (2005).

3) Ross S. A., Gulve E. A., Wang M., Chem. Rev., 104, 1255-1282 (2004).

4) Kumar G. S., Tiwari A. K., Rao V. R., Prasad K. R., Ali A. Z., Babu K. S., J. Asian Nat. Prod. Res., 12, 978-984 (2010).

5) Takahashi T., Miyazawa M., Med. Chem. Res., 21, 1762-1770 (2012).

6) Wang Y., Xiang L., Wang C., Tang C., He X., PLoS ONE, 8, e71144
}

(2013).

7) Raju B. C., Tiwari A. K., Kumar J. A., Ali A. Z., Agawane S. B., Saidachary G., Madhusudana K., Bioorg. Med. Chem., 18, 358-365 (2010).

8) Ranga Rao R., Tiwari A. K., Prabhakar Reddy P., Suresh Babu K., Ali A. Z., Madhusudana K., Madhusudana Rao J., Bioorg. Med. Chem., 17, 5170-5175 (2009).

9) Yang D., Xie H., Jiang Y., Wei X., Food Chem., 194, 857-863 (2016).

10) Lin L. G., Liu Q. Y., Ye Y., Planta Med., 80, 1053-1066 (2014).

11) Yan J., Sun L. R., Zhou Z. Y., Chen Y. C., Zhang W. M., Dai H. F., Tan J. W., Phytochemistry, 80, 37-41 (2012).

12) Siddaiah V., Maheswara M., Venkata Rao C., Venkateswarlu S., Subbaraju G. V., Bioorg. Med. Chem. Lett., 17, 1288-1290 (2007).

13) Lin L. G., Xie H., Li H. L., Tong L. J., Tang C. P., Ke C. Q., Liu Q. F., Lin L. P., Geng M. Y., Jiang H., Zhao W. M., Ding J., Ye Y., J. Med. Chem., 51, 4419-4429 (2008).

14) Famuyiwa S. O., Ntumy A. N., Andrae-Marobela K., Yeboah S. O., S. Afr. J. Bot., 88, 17-22 (2013).

15) Emami S., Ghanbarimasir Z., Eur. J. Med. Chem., 93, 539-563 (2015).

16) Noushini S., Alipour E., Emami S., Safavi M., Ardestani S. K., Gohari A. R., Shafiee A., Foroumadi A., Daru, 21, 31 (2013).

17) Perjési P., Das U., De Clercq E., Balzarini J., Kawase M., Sakagami H., Stables J. P., Lorand T., Rozmer Z., Dimmock J. R., Eur. J. Med. Chem., 43, 839-845 (2008).

18) Letafat B., Shakeri R., Emami S., Noushini S., Mohammadhosseini N., Shirkavand N., Kabudanian Ardestani S., Safavi M., Samadizadeh M., Letafat A., Sha Ee A., Foroumadi A., Iran. J. Basic Med. Sci., 16, 1155-1162 (2013).

19) Roy S. K., Kumari N., Gupta S., Pahwa S., Nandanwar H., Jachak S. M., Eur. J. Med. Chem., 66, 499-507 (2013).

20) Siddaiah V., Rao C. V., Venkateswarlu S., Krishnaraju A. V., Subbaraju G. V., Bioorg. Med. Chem., 14, 2545-2551 (2006).

21) Foroumadi A., Samzadeh-Kermani A., Emami S., Dehghan G. Sorkhi M., Arabsorkhi F., Heidari M. R., Abdollahi M., Shafiee A., Bioorg. Med. Chem. Lett., 17, 6764-6769 (2007).

22) Jacquot Y., Byrne C., Xicluna A., Leclercq G., Med. Chem. Res., 22 681-691 (2013).

23) Desideri N., Bolasco A., Fioravanti R., Monaco L. P., Orallo F. Yáñez M., Ortuso F., Alcaro S., J. Med. Chem., 54, 2155-2164 (2011).

24) Gan C., Zhao Z., Nan D. D., Yin B., Hu J., Eur. J. Med. Chem., 76, 125-131 (2014).

25) Yoshikawa M., Shimada H., Nishida N., Li Y., Toguchida I., Yamahara J., Matsuda H., Chem. Pharm. Bull., 46, 113-119 (1998).

26) Kim J. S., Kwon Y. S., Sa Y. J., Kim M. J., J. Agric. Food Chem., 59, 138-144 (2011).

27) Desideri N., Olivieri S., Stein M. L., Orsi N., Conti C., Antivir. Chem. Chemother., 8, 545-555 (1997).

28) Cheng X. M., Huang Z. T., Zheng Q. Y., Tetrahedron, 67, $9093-$ 9098 (2011).

29) Sangwan N. K., Rastogi S. N., Indian J. Chem., 19B, 500-503 (1980).

30) Li Y. F., Liu Z. Q., Luo X. Y., J. Agric. Food Chem., 58, 4126-4131 (2010).

31) Nile S. H., Kim S. H., Ko E. Y., Park S. W., Biomed. Res. Int., 2013 , 718065 (2013)

32) Takahashi T., Miyazawa M., Med. Chem. Res., 21, 1762-1770 (2012). 